\title{
Efficacy of high resolution computed tomography for detection of early healing in scaphoid fractures
}

\author{
Michael Di Ianni, Jatin Kaicker, Ke Wu, Hema Choudur* \\ Department of Medical Imaging, McMaster University, Hamilton, Canada \\ Email: *choudur@hhsc.ca
}

Received 18 April 2012; revised 25 May 2012; accepted 20 June 2012

\begin{abstract}
Background: While plain radiography is commonly used to assess scaphoid fracture, this imaging modality may not accurately demonstrate early bone healing. This investigation evaluates the utility of 64-slice CT in the detection of early fracture healing compared to plain radiographs and magnetic resonance imaging (MRI). Methods: Outpatients attending follow-up visits for scaphoid fractures at Hamilton General Hospital were included in this investigation. Inclusion criteria included outpatients over age of 18 who consented to attend a follow-up visit for the scaphoid fracture at 6 weeks for X-Ray, 64-slice CT and MRI scan to monitor fracture healing. Assessment of healing was independently interpreted by two radiologists specialized in musculoskeletal imaging. A total of 7 adult outpatients were accrued, with each case classified as healing, not healing, or equivocal for plain radiography and 64-slice CT scan. Results: For plain radiographs, the level of interrater agreement for evidence of healing was $57 \%(4 / 7)$ cases. When comparing this to CT scans, there was no discrepancy between radiologists as $100 \%(7 / 7)$ were found to have evidence of healing. The 64-slice CT scan demonstrated evidence of early trabecular continuity in all cases, including radiographs that were interpreted as equivocal. Conclusion: This study can be considered a pilot project for the efficacy of 64-slice CT in the assessment of early healing of scaphoid fractures. For clinicians, the multiplanar reconstruction images allows for more accurate assessment of fractures than plain radiography. CT scans are able to penetrate through bony callus that may obscure visualization of healing in plain radiography, demonstrate trabecular continuity better than plain radiographs, are readily accessible and provide faster imaging acquisition. The observations from this study may have implications in terms of duration and type of casting applied, timing of strengthening exercises, and avoiding unnecessary surgery which affect patient morbidity and cost of care.
\end{abstract}

${ }^{*}$ Corresponding author.
Keywords: Wrist Fracture; Scaphoid Bone Fracture; Scaphoid Bone Fracture Healing; 64-Slice CT Scans; Trabecular Continuity

\section{INTRODUCTION}

Scaphoid fractures are a common injury that may lead to considerable morbidity, accounting for about 50\% - 80\% of carpal injuries [1]. They have an incidence of 38/ 100,000 in men and 8/100,000 in females [2]. The fractures are classified according to stability, location and displacement of the fracture. These fractures can sometimes be diagnosed as simple wrist sprains, and if left untreated develop into a malunion or nonunion and diminished grip strength and range of motion [2].

Diagnosis of a scaphoid fracture depends on physical examination of the wrist and plain radiographs at the time of injury. First line investigation includes plain radiography including dedicated scaphoid views. If there is clinical suspicion of a scaphoid fracture but plain radiographs are negative, other imaging modalities such as CT or MRI can be used. Studies have found that CT and MRI are both effective at diagnosing scaphoid fractures with an accuracy of $91 \%$ versus $85 \%$ respectively [3].

The treatment of the fractures is dependent on its type. Nondisplaced fractures are treated with long-arm thumb spica cast with the wrist in neutral position for 6 weeks, followed by a short-arm spica cast for an additional 6 weeks if radiographs depict early bony union. Displacement or widening of the fracture at this point is managed surgically. Moreover, displaced fractures require open reduction and internal fixation with Kirschner wires or a Herbert screw and four to six months of rehabilitation [4].

Once a scaphoid fracture is identified, it is followed every six weeks for progression of healing or complications to prevent morbidity [5]. For the patient, one of the major complications is avascular necrosis of the scaphoid bone due to lack of retrograde blood supply, which is seen in approximately $30 \%-40 \%$ of cases and is more common in proximal fractures [5]. An inability to diag- 
nose a non-healing fracture may also lead to degenerative arthritis, especially at the radiocarpal joint. This can result in a limited range of motion and grip strength in patients. Economically, the ability to determine a non-healing fracture can lead to loss of daily function and workplace productivity, and the added healthcare costs of rehabilitation for these patients [5]. Similarly, if healing cannot be clearly visualized, the patient remains in a splint for an additional 6 weeks, causing discomfort to the patient, requiring further time off from work, and adding to the healthcare cost needed for additional, unnecessary follow-up.

An important issue with the management of scaphoid fracture is that standard radiographs taken at 6 weeks post injury may not accurately demonstrate early bone healing as the overlying cast can obscure the fracture, affecting management decisions. The three dimensional shape of the scaphoid hinders the evaluation of the fracture location. The anteroposterior views used, even when taken with the wrist in the positon of ulnar deviation, results in images that are distorted by the flexion and normal curvature of the scaphoid. This pilot investigation evaluates the utility of 64-slice CT in the detection of early fracture healing as compared to plain radiographs and magnetic resonance imaging (MRI). Thus, the objective of this investigation is to stimulate discussion of radiologists, orthopaedic surgeons and residents regarding the use of high-resolution 64-slice computed tomography (CT) in the follow-up of scaphoid fractures.

\section{METHODS}

Patients were included in this investigation if they were over the age of 18, presented with a scaphoid fracture and attended a follow-up visit at Hamilton General Hospital. The 6 week follow-up visit was selected as it is common practice for scaphoid fracture patients to be seen for potential bone healing. After explaining the risk of the excess radiation from the CT scan, patients who consented were included. Hospital inpatients or outpatients who presented to the radiology clinic for a wrist fracture that was not related to the scaphoid were excluded. If a patient opted to forgo a 6 week follow-up or visit an additional medical institution, they were not considered for the investigation.

All radiological images were independently interpreted by two radiologists specialized in musculoskeletal imaging. They interpreted the studies in a random order and were blinded to any previous interpretation and to the patient's identity. For this investigation, a total of 7 adult outpatients were accrued. Six weeks after initial identification and management of the fracture, they had follow-up radiographs, 64-slice CT, and MRI to monitor fracture healing. Each case was classified as healing, not healing, or equivocal.

\section{RESULTS}

For patients presenting with a scaphoid fracture, anteroposterior (AP) view plain radiographs of the wrist were taken upon initial presentation and at 6 week follow-up were assessed (Figure 1). Obscuration of the fracture line, callus formation and cortical continuity were evaluated as features to suggest healing. Furthermore, two millimeter axial Computer Tomography (CT) slices of the wrist with sagittal and coronal reformations were obtained (Figure 2). Trabecular continuity across the fracture was assessed as the major factor implying early bone healing on CT. In determining a cutoff positive healing versus non-healing, 5 or more bridging trabeculae was considered. For all included patients, an MRI was performed. The MRI included limited coronal T1, short T1 Inversion recovery (STIR) and double-echo steady state (DESS) sequences to assess bone marrow edema and cortical continuity (Figure 3 ).

Two musculoskeletal radiologists from Hamilton General Hospital assessed the patients using plain radiography, CT scans and MRI at 6 weeks for evidence of healing. Using plain radiographs, of the cases seen, there was agreement for evidence of healing in 57\% (4/7) cases.

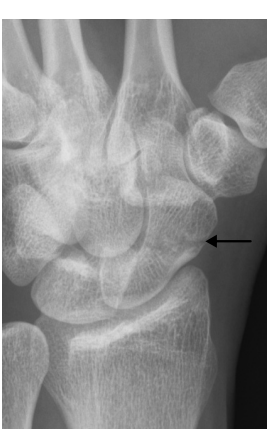

At time of injury

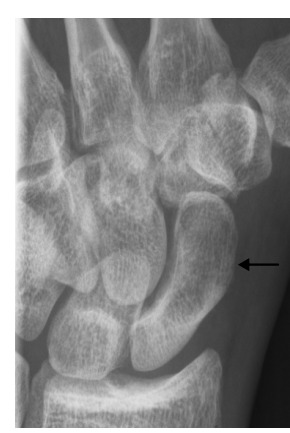

6 week follow-up
Figure 1. Assessing scaphoid fracture at time of injury and 6 week follow-up using plain radiography. Note the undisplaced fracture of the midscaphoid at the time of injury with obscuration of the fracture line on follow-up 6 week suggestive of early fracture healing.
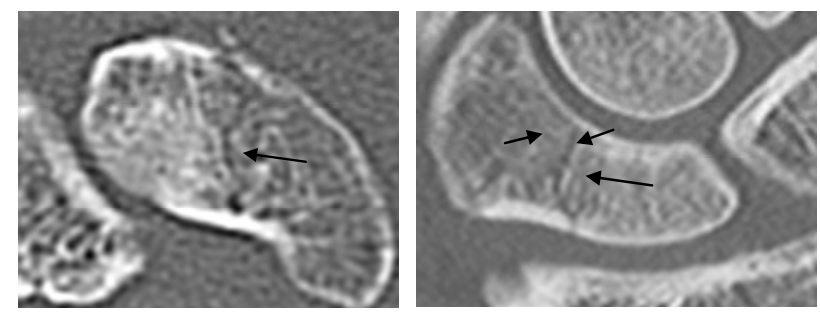

Figure 2. $2 \mathrm{~mm}$ axial CT slices of the wrist with sagittal and coronal reformations demonstrating fracture healing. Arrows depict the trabecular continuity across the fractures. Cortical break along the fracture is clearly depicted on the CT and not visualized on plain radiographs. 


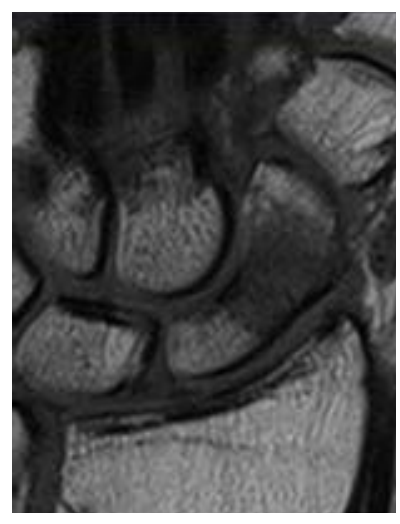

T1

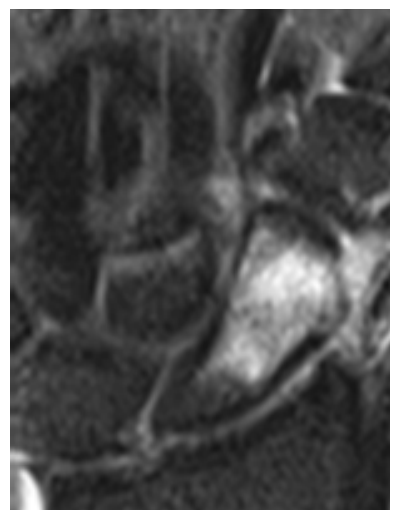

Short T2 Inversion (STIR) recovery

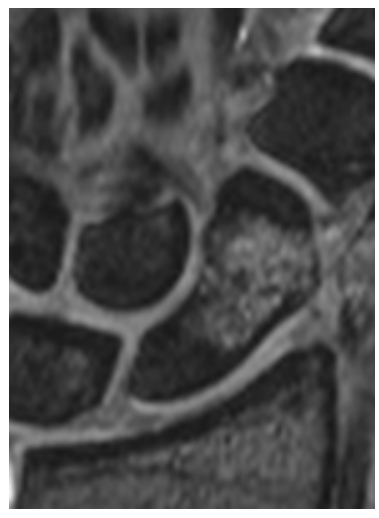

Double-echo steady state (DESS)

Figure 3. Assessing bone marrow edema and cortical continuity using Magnetic Resonance Imaging (MRI): The scaphoid fracture is faintly demonstrated on the coronal MR DESS sequence as a linear low T1 signal (arrow). The intense surrounding bone marrow edema appears hypointense on T1 and hyperintense on STIR and gradient DESS sequences.

CT scan assessment at 6 weeks demonstrated no discrepancy between the two radiologists as $100 \%(7 / 7)$ were found to have evidence of healing (Table 1). Thus, the 64-slice CT scan demonstrated unequivocal evidence of early trabecular continuity in all cases, including those in which radiographs were interpreted as equivocal. While MRI scans were acquired for every patient in the investigation, the images were inconclusive and noncontributory in all cases for assessment of trabecular continuity.

\section{DISCUSSION}

For patients presenting with scaphoid fractures, the ability to distinguish between healing and complications is imperative to avoid complications such as avascular necrosis. While it is important to accurately diagnose fractures in patients and prescribe a treatment regime, overtreatment can have adverse implications due to the implication on the patient's quality of life with a restrictive cast and the additional cost of treatment. Accurate diagnosis of healing at the 6 week mark is essential to ensure a patient centered treatment plan to help individuals regain func- tion of their wrist.

In this study, using 6 week follow-up plain radiography, the two radiologists were in agreement in only 57\% of the cases. This is consistent with previous literature that plain radiography is useful for immediate assessment of wrist injury, but has a low sensitivity in examining follow-up of scaphoid fractures [6]. In our study, the CT scans demonstrated unequivocal evidence of early trabecular continuity in all the 6 week follow-up scaphoid fractures, including those cases in which plain radiographs were interpreted as equivocal. 64-slice CT scans offers greater inter-observer agreement as seen in our study with an agreement of $100 \%$ between the two musculoskeletal radiologists. While MRI scans were acquired for every patient in the investigation, the images were inconclusive and non-contributory for assessment of trabecular continuity. MRI primarily is used for depicting early occult scaphoid fractures by demonstrating the fracture and surrounding marrow edema. MRI is also useful for demonstration of bone contusion in the absence of a fracture, when the patient has tenderness in the anatomical snuff box at 3 - 6 weeks follow-up.

Table 1. Comparison of plain radiograph and CT scan scaphoid fracture healing assessment.

\begin{tabular}{|c|c|c|c|c|}
\hline \multirow[b]{2}{*}{ Patient ID } & \multicolumn{2}{|c|}{ Radiologist 1} & \multicolumn{2}{|c|}{ Radiologist 2} \\
\hline & Plain radiography at 6 weeks & CT Scan at 6 weeks & Plain radiography at 6 weeks & CT Scan at 6 weeks \\
\hline A & Evidence of healing & Evidence of healing & Evidence of healing & Evidence of healing \\
\hline $\mathrm{B}$ & Evidence of healing & Evidence of healing & Equivocal evidence & Evidence of healing \\
\hline $\mathrm{C}$ & Equivocal evidence & Evidence of healing & Evidence of healing & Evidence of healing \\
\hline $\mathrm{D}$ & Evidence of healing & Evidence of healing & Equivocal evidence & Evidence of healing \\
\hline $\mathrm{E}$ & Evidence of healing & Evidence of healing & Evidence of healing & Evidence of healing \\
\hline $\mathrm{F}$ & Evidence of healing & Evidence of healing & Evidence of healing & Evidence of healing \\
\hline G & Evidence of healing & Evidence of healing & Evidence of healing & Evidence of healing \\
\hline
\end{tabular}


This study demonstrates several advantages of the multi-detector, thin slice, high resolution CT in the assessment of scaphoid fractures at 6 weeks follow-up. For clinicians, the multiplanar reconstruction images with their superior spatial resolution allow for more clear and accurate demonstration of the fracture than plain radiography, and allows for better visualization of the trabeculae and their continuity. CT is able to provide better visualization of the fracture including sharp delineation of the cortex and trabeculae when the cast obscures bony details on plain radiography. CT scans also have the ability to better assess other structures in the region including other carpal bones and adjacent soft tissues and can potentially pick up co existing abnormality that is occult on plain radiography. In comparison to plain radiography, CT scans are able to penetrate through bony callus that may obscure the visualization of healing on plain radiography. CT can also differentiate the trabecular pattern better than MRIs. MRIs have been shown to possess better diagnostic performance for detection of occult scaphoid fractures, with added soft tissue evaluation, but are expensive and do not contribute to assessment of early fracture healing and trabecular continuity [6]. From the patient's perspective, CT scans are more accessible, provide faster imaging acquisition, limit patient discomfort and have fewer contraindications (pacemakers) than MRI.

While there are many advantages to multi-detector, thin slice, high resolution CT scanning in assessing scaphoid fracture healing, there are some disadvantages. The first is that a higher radiation dose is needed than plain radiography. This drawback can be addressed by exposing only the area of interest to the radiation. Appropriate use of lead shielding, and having the patients stretch their arms above their head to limit the dose to the rest of the body can limit total body radiation exposure. The added limitation is the increased costs and reduced availability of CT scanners compared to plain radiography though is commonplace throughout hospitals in Canada and many countries worldwide. The increased cost of this investigation can be offset economically by definite early determination of fracture healing or lack of healing in follow-up patients. For patients with definitive healing, as determine by the CT scan, morbidity can be reduced as they can return to work earlier by employing a short arm spica cast. For athletes in particular, CT assessment can allow for early onset exercises and decisions regarding avoidance of surgical intervention for the fracture.

The main strength of this pilot project lies in the novel approach of utilizing the 64-slice CT for definitive and unequivocal assessment of early scaphoid fracture healing by evaluating trabecular continuity as compared to the routine practice of follow-up plain radiography. The main limitation of this investigation was the sample size. The intention of the investigators was to include 12 - 15 patients and analyze the results with chi-square and one way analysis of variance methods. Due to the small sample accrued, a meaningful statistical analysis could not be performed.

\section{CONCLUSION}

This study can be considered a pilot project for the efficacy of 64-slice CT in the assessment of early healing of scaphoid fractures by evaluating trabecular continuity as compared to plain radiographs, despite the limiting factor of a small sample size. Trabeculae across the fracture are clearly and unequivocally demonstrated by 64-slice CT unlike plain radiographs given the advantages of thin slice thickness, multiplanar reformatting ability and optimum penetration through the overlying cast and callus. Though not assessed on this study, 64-slice CT also demonstrates cortical healing and obscuration of the fracture more accurately than follow-up plain radiography, thus affecting management decisions. 64-slice CT shows promise in demonstration of early fracture healing and can be used as replacement for follow-up plain radiography in athletes to reduce morbidity and hasten return to sports. It can also be used when follow-up plain radiography is equivocal and hinders management decisions. The observations from this pilot study may have implications in terms of the duration and type of casting applied, timing of strengthening exercises, and avoiding unnecessary surgery; all of which affect patient morbidity, quality of life, and cost of care.

\section{ACKNOWLEDGEMENTS}

The authors would like to thank the Department of Medical Imaging at Hamilton General Hospital for supporting this project.

\section{REFERENCES}

[1] Hackney, L.A. and Dodds, S.D. (2011) Assessment of scaphoid fracture healing. Current Reviews in Musculoskeletal Medicine, 4, 16-22. doi:10.1007/s12178-011-9072-0

[2] Hickey, B., Hak, P. and Logan, A. (2012) Review of treatment of acute scaphoid fractures: R1. Australia and New Zealand Journal of Surgery, 82, 118-121. doi:10.1111/j.1445-2197.2012.06000.x

[3] Mallee, W., Doornberg J.N., Ring, D., Van Dijk, C.N., Maas, M. and Goslings, J.C. (2011) Comparison of CT and MRI for diagnosis of suspected scaphoid fractures. The Journal of Bone and Joint Surgery, 93, 20-28. doi:10.2106/JBJS.I.01523

[4] Dias, J.J., Wildin, C.J., Bhowal, B. and Thompson, J.R. (2005) Should acute scaphoid fractures be fixed? A randomized controlled trial. The Journal of Bone and Joint 
Surgery, 87, 2160-2168. doi:10.2106/JBJS.D.02305

[5] Hunter, J.M., Mackin, E.J. and Callahan, A.D. (1995) Rehabilitation of the hand: Surgery and therapy. 4th Edition, Mosby-Year Book, Philadelphia.
[6] Jenkins, P.J., Slade, K., Huntley, J.S. and Robinson, C.M. (2008) A comparative analysis of the accuracy, diagnostic uncertainty and cost of imaging modalities in suspected scaphoid fractures. Injury, 39, 768-774. 